\title{
AN IMPROVED AND SIMPLIFIED FULL-NEWTON STEP $O(n)$ INFEASIBLE INTERIOR-POINT METHOD FOR LINEAR OPTIMIZATION*
}

\author{
C. $\operatorname{ROOS}^{\dagger}$
}

\begin{abstract}
We present an improved version of an infeasible interior-point method for linear optimization published in 2006. In the earlier version each iteration consisted of one so-called feasibility step and a few - at most three - centering steps. In this paper each iteration consists of only a feasibility step, whereas the iteration bound improves the earlier bound by a factor $2 \sqrt{2}$. The improvements are due to a new lemma that gives a much tighter upper bound for the proximity after the feasibility step.
\end{abstract}

Key words. linear optimization, interior-point method, infeasible method, primal-dual method, polynomial complexity

AMS subject classifications. 90C05, 90C51

DOI. $10.1137 / 140975462$

1. Introduction. It is now well-understood that the success of interior-point methods (IPMs) is due to the fact that the iterates move through the interior of the feasible region while staying away from the boundary. In the analysis of feasible IPMs this is achieved by staying close to the central path of the problem, which is a smooth curve in the interior of the feasible region that serves as a guide to the set of optimal optimal solutions. A feasible IPM can be started only if a strictly feasible point is known. Usually such a starting point is not at hand. In that case a so-called infeasible IPM (IIPM) should be used.

There exists a wide variety of IIPMs. See, e.g., [1, 3, 5, 6, 7, 8, 9, 12, 14]. Many of these methods start with a strictly feasible solution of an artificially constructed problem, and they generate iterates each of which is strictly feasible for that or another artificial problem. Eventually the artificial problem converges to the problem that has to be solved and the iterates converge to an optimal solution of that problem.

So in IIPMs each of the iterates is strictly feasible for some (intermediate) artificial problem and, moreover, depending on the method stay "close" or "not so close" to the central path of that problem, where closeness is measured by some merit function. The methods with the best theoretical performance belong to the first category: their iterates closely follow some homotopy path. The method in this paper also falls within this category. It is a simplified and improved version of the method in [10], where we presented the first full-Newton step $O(n)$ IIPM for linear optimization (LO). For a more detailed discussion of IIPMs and a motivation for using full-Newton step methods we refer to [7] and [10], respectively.

The basis for this paper is Lemma A.1 in Appendix A. It enables us to get a much tighter bound for the proximity measure after a full Newton step than in [10]. In the terminology of [10] it means that after a feasibility step the new iterates are sufficiently centered. As a consequence each (main) iteration in the new algorithm

\footnotetext{
${ }^{*}$ Received by the editors July 1, 2014; accepted for publication (in revised form) October 20, 2014; published electronically January 8, 2015.

http://www.siam.org/journals/siopt/25-1/97546.html

${ }^{\dagger}$ Faculty of Electrical Engineering, Computer Science and Mathematics, Delft University of Technology, P.O. Box 5031, 2600 GA Delft, The Netherlands (C.Roos@tudelft.nl).
} 
needs only one feasibility step, whereas the previous algorithm needed three additional centering steps in each (main) iteration. Besides this the use of Lemma A.1 also leads to a simpler analysis.

As usual, we consider the LO problem in the standard form

$$
\min \left\{c^{T} x: A x=b, x \geq 0\right\}
$$

with its dual problem

$$
\max \left\{b^{T} y: A^{T} y+s=c, s \geq 0\right\} .
$$

Here $A \in \mathbf{R}^{m \times n}, b, y \in \mathbf{R}^{m}$, and $c, x, s \in \mathbf{R}^{n}$. Without loss of generality we assume that $\operatorname{rank}(A)=m$.

As in [10], and in all other papers on IIPMs, it is assumed that there exists an optimal solution $\left(x^{*}, y^{*}, s^{*}\right)$ such that $\left\|\left(x^{*} ; s^{*}\right)\right\|_{\infty} \leq \zeta$. In our algorithm the initial iterates will be $\left(x^{0}, y^{0}, s^{0}\right)=\zeta(e, 0, e)$. Note that since $x^{*}$ and $s^{*}$ are nonnegative and have inner product zero, $\left\|\left(x^{*} ; s^{*}\right)\right\|_{\infty} \leq \zeta$ holds if and only if

$$
0 \leq x^{*} \leq \zeta e, \quad 0 \leq s^{*} \leq \zeta e .
$$

The outline of the paper is as follows. In section 2 we briefly recall some material from [10] that we need in this paper and also present the new algorithm. Section 3 contains the analysis of the new algorithm. In section 3.1 we deviate from [10] by giving a much tighter upper bound for the proximity measure after a step. This result depends on Lemma A.1 in the appendix and expresses this bound in terms of a quantity $\omega(v)$. One should note that the definition of $\omega(v)$ in this paper slightly differs from $\omega(v)$ in [10]. Sections 3.2, 3.3, and 3.4 serve to derive an upper bound for $\omega(v)$. In these sections there is some overlap with the corresponding sections in [10], but since we changed the definition of $\omega(v)$ we included these sections, also to make the paper self-contained. In section 3.5 we fix values for the parameters $\theta$ and $\tau$ in the algorithm. Here $\tau$ is a uniform upper bound for the values of the proximity measure $\delta(x, s ; \mu)$ occurring during the course of the algorithm, and $\theta$ determines the progress to feasibility and optimality of the iterates. As a result we obtain that the algorithm is well defined for the chosen values of $\theta$ and $\tau$, provided that $n \geq 2$. Finally, section 4 contains some concluding remarks.

2. Infeasible full-Newton step IPM. In the case of an infeasible method we call the triple $(x, y, s)$ an $\varepsilon$-solution of $(\mathrm{P})$ and $(\mathrm{D})$ if the 2-norms of the residual vectors $b-A x$ and $c-A^{T} y-s$ do not exceed $\varepsilon$, and also $x^{T} s \leq \varepsilon$. In this section we present an infeasible-start algorithm that generates an $\varepsilon$-solution of (P) and (D), if it exists, or establishes that no such solution exists.

2.1. The perturbed problems. We start with choosing arbitrarily $x^{0}>0$ and $y^{0}, s^{0}>0$ such that $x^{0} s^{0}=\mu^{0} e$ for some (positive) number $\mu^{0}$. For any $\nu$ with $0<\nu \leq 1$ we consider the perturbed problem $\left(\mathrm{P}_{\nu}\right)$, defined by

$$
\min \left\{\left(c-\nu\left(c-A^{T} y^{0}-s^{0}\right)\right)^{T} x: A x=b-\nu\left(b-A x^{0}\right), \quad x \geq 0\right\},
$$

and its dual problem $\left(\mathrm{D}_{\nu}\right)$, which is given by

$$
\max \left\{\left(b-\nu\left(b-A x^{0}\right)\right)^{T} y: A^{T} y+s=c-\nu\left(c-A^{T} y^{0}-s^{0}\right), \quad s \geq 0\right\} .
$$


The same pair of perturbed problems has been studied in [7]. In that paper the authors show that many IIPMs are realizations of their prototype IIPM, in which the intermediate artificial problems have the form of the perturbed problems $\left(\mathrm{P}_{\nu}\right)$ and $\left(\mathrm{D}_{\nu}\right)$. They demonstrate this explicitly for the IIPMs in, e.g., $[4,9,13]$ The method discussed in this paper is different in the sense that we do not need line searches, because the step size is always 1 . Since the search direction is the usual Newton step, as in [7], we call our method a full-Newton step method.

Note that if $\nu=1$, then $x=x^{0}$ yields a strictly feasible solution of $\left(\mathrm{P}_{\nu}\right)$ and $(y, s)=\left(y^{0}, s^{0}\right)$ a strictly feasible solution of $\left(\mathrm{D}_{\nu}\right)$. We conclude that if $\nu=1$, then $\left(\mathrm{P}_{\nu}\right)$ and $\left(\mathrm{D}_{\nu}\right)$ are strictly feasible, i.e., they satisfy the interior point condition (IPC).

Without proof we recall a result that follows from a slightly more general result in $[7$, section 3].

LEMMA 2.1. The problems $(\mathrm{P})$ and $(\mathrm{D})$ are feasible if and only if the perturbed problems $\left(\mathrm{P}_{\nu}\right)$ and $\left(\mathrm{D}_{\nu}\right)$ satisfy the IPC for every $\nu$ satisfying $0<\nu \leq 1$.

In what follows we assume that $(\mathrm{P})$ and $(\mathrm{D})$ are feasible. It may be worth noting that if $x^{0}$ and $\left(y^{0}, s^{0}\right)$ are feasible, then $\left(\mathrm{P}_{\nu}\right) \equiv(\mathrm{P})$ and $\left(\mathrm{D}_{\nu}\right) \equiv(\mathrm{D})$ for each $\nu \in(0,1]$.

2.2. The central path of the perturbed problems. Let $(\mathrm{P})$ and $(\mathrm{D})$ be feasible and $0<\nu \leq 1$. Then Lemma 2.1 implies that the problems $\left(\mathrm{P}_{\nu}\right)$ and $\left(\mathrm{D}_{\nu}\right)$ satisfy the IPC, and hence their central paths exist. This means that the system

$$
\begin{array}{rlrl}
b-A x & =\nu\left(b-A x^{0}\right), & x \geq 0, \\
c-A^{T} y-s & =\nu\left(c-A^{T} y^{0}-s^{0}\right), & & s \geq 0, \\
x s & =\mu e & &
\end{array}
$$

has a unique solution for every $\mu>0$. This solution consists of the $\mu$-centers of the perturbed problems $\left(\mathrm{P}_{\nu}\right)$ and $\left(\mathrm{D}_{\nu}\right)$. In what follows the parameters $\mu$ and $\nu$ always satisfy the relation $\mu=\nu \mu^{0}$.

2.3. An iteration of our algorithm. We just established that if $\nu=1$ and $\mu=\mu^{0}$, then $x=x^{0}$ is the $\mu$-center of the perturbed problem $\left(\mathrm{P}_{\nu}\right)$ and $(y, s)=\left(y^{0}, s^{0}\right)$ the $\mu$-center of $\left(\mathrm{D}_{\nu}\right)$. As stated before the initial iterates are given by $\left(x^{0}, y^{0}, s^{0}\right)=$ $\zeta(e, 0, e)$, where $\zeta$ satisfies (1.1) for some optimal solutions $\left(x^{*}, y^{*}, s^{*}\right)$ of $(\mathrm{P})$ and $(\mathrm{D})$. Moreover $\mu^{0}=\zeta^{2}$.

If the triple $(x, y, s)$ is feasible for the problem pair $\left(\mathrm{P}_{\nu}\right)$ and $\left(\mathrm{D}_{\nu}\right)$, and $\mu=\nu \zeta^{2}$, then we measure proximity to the $\mu$-center of this perturbed problem pair by the quantity

$$
\delta(x, s ; \mu):=\delta(v):=\frac{1}{2}\left\|v-v^{-1}\right\|, \quad \text { where } \quad v:=\sqrt{\frac{x s}{\mu}} .
$$

As an immediate consequence we have the following simple result.

Lemma 2.2 (see [11, Lemma II.62]). With $\delta=\delta(v)$, one has $\sqrt{1+\delta^{2}}-\delta \leq v_{i} \leq$ $\sqrt{1+\delta^{2}}+\delta$, for each $i$.

Initially we have $\delta(x, s ; \mu)=\delta\left(x^{0}, s^{0} ; \mu^{0}\right)=0$. In what follows we assume that at the start of each iteration, just before the $\mu$-update, $\delta(x, s ; \mu)$ is smaller than or equal to a (small) threshold value $\tau>0$. So this is certainly true at the start of the first iteration.

Now we briefly describe one (main) iteration of our algorithm. Suppose that for some $\mu \in\left(0, \mu^{0}\right]$ we have $x, y$, and $s$ satisfying the feasibility conditions (2.1) and (2.2) for $\nu=\mu / \mu^{0}$ and such that $\delta(x, s ; \mu) \leq \tau$. We reduce $\mu$ to $\mu^{+}=(1-\theta) \mu$, with 
$\theta \in(0,1)$, and find new iterates $x^{+}, y^{+}$, and $s^{+}$that satisfy (2.1) and (2.2), with $\mu$ replaced by $\mu^{+}$and $\nu$ by $\nu^{+}=\mu^{+} / \mu^{0}$, and such that $\delta\left(x^{+}, s^{+} ; \mu^{+}\right) \leq \tau$. Note that $\nu^{+}=(1-\theta) \nu$. So the relation $\mu=\nu \mu^{0}=\nu \zeta^{2}$ is maintained in every iteration.

We proceed by describing the search direction in the algorithm. It is the same as the so-called feasibility direction in [10], namely, the (unique) solution of the following system:

$$
\begin{aligned}
A \Delta x & =\theta \nu r_{b}^{0}, \\
A^{T} \Delta y+\Delta s & =\theta \nu r_{c}^{0}, \\
s \Delta x+x \Delta s & =\mu e-x s,
\end{aligned}
$$

where $r_{b}^{0}$ and $r_{c}^{0}$ denote the initial values of the primal and dual residuals:

$$
r_{b}^{0}=b-A x^{0}, \quad r_{c}^{0}=c-A^{T} y^{0}-s^{0} .
$$

After a full-Newton step the iterates are denoted as

$$
x^{+}=x+\Delta x, \quad y^{+}=y+\Delta y, \quad s^{+}=s+\Delta s .
$$

As we showed in [10], these iterates satisfy the affine equations in (2.1) and (2.2) with $\nu=\nu^{+}$. The hard part in the analysis is to guarantee that $x^{+}$and $s^{+}$are positive and satisfy $\delta\left(x^{+}, s^{+} ; \mu^{+}\right) \leq \tau$.

2.4. The algorithm. A formal description of the new algorithm is given in Figure 1.

Recall from [10] that after each iteration the residual vectors are reduced by a factor $1-\theta$. The algorithm stops if the norms of the residuals and the duality gap are less than the accuracy parameter $\varepsilon$.

Primal-Dual InfEasible IPM

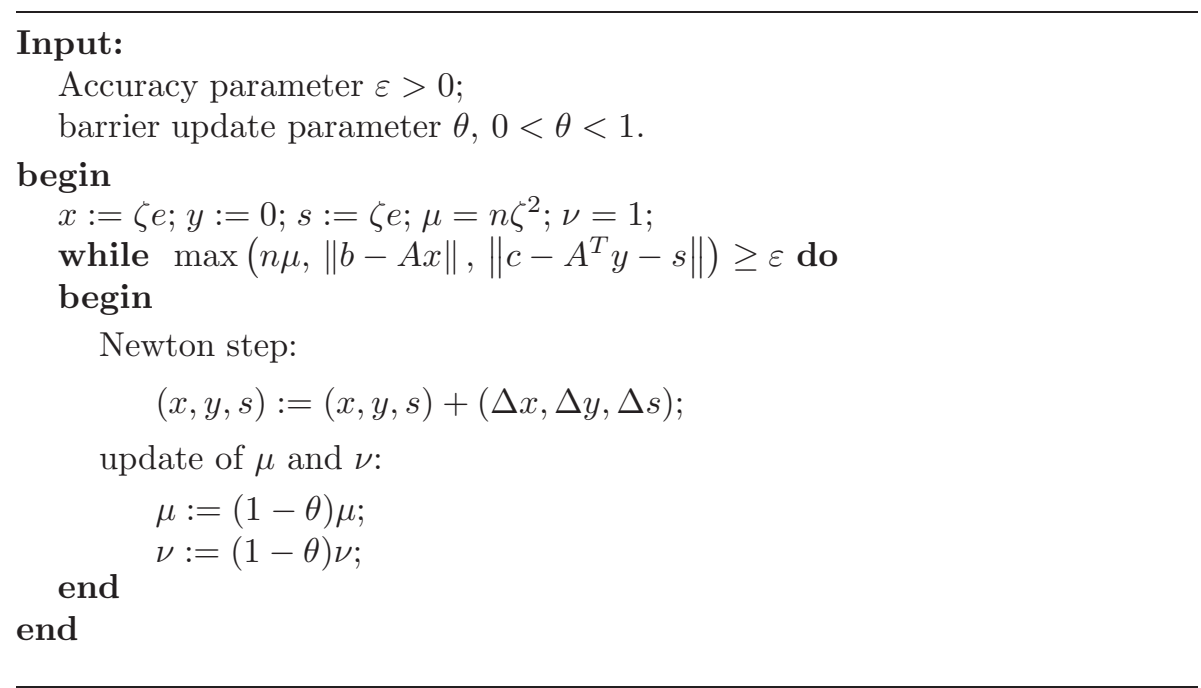

FIG. 1. The algorithm.

Copyright (C) by SIAM. Unauthorized reproduction of this article is prohibited. 
3. Analysis of the algorithm. Let $x, y$, and $s$ denote the iterates at the start of an iteration, and assume $\delta(x, s ; \mu) \leq \tau$.

3.1. Upper bound for $\delta\left(\boldsymbol{v}^{+}\right)$. As we established in section 2.3, the Newton step generates new iterates $\left(x^{+}, y^{+}, s^{+}\right)$that satisfy the feasibility conditions for $\left(\mathrm{P}_{\nu^{+}}\right)$ and $\left(\mathrm{D}_{\nu^{+}}\right)$, except possibly the nonnegativity constraints. A crucial element in the analysis is to show that after the Newton step $\delta\left(x^{+}, s^{+} ; \mu^{+}\right) \leq \tau$. We define the scaled search directions $d_{x}$ and $d_{s}$ as follows:

$$
d_{x}:=\frac{v \Delta x}{x}, \quad d_{s}:=\frac{v \Delta s}{s},
$$

Using (2.7) and (3.1) one may easily verify that $x^{+} s^{+}=\mu\left(e+d_{x} d_{s}\right)$.

LEMma 3.1 (see [11, Lemma II.48]). The Newton step is strictly feasible if and only if $e+d_{x} d_{s}>0$.

Corollary 3.2. The iterates $\left(x^{+}, y^{+}, s^{+}\right)$are strictly feasible if $\left\|d_{x} d_{s}\right\|_{\infty}<1$.

Proof. By Lemma 3.1, $x^{+}$and $s^{+}$are strictly feasible if and only if $e+d_{x} d_{s}>0$. Since the last inequality holds if $\left\|d_{x} d_{s}\right\|_{\infty}<1$, the corollary follows.

In what follows we use the notation $\omega(v):=\frac{1}{2}\left(\left\|d_{x}\right\|^{2}+\left\|d_{s}\right\|^{2}\right)$ and we assume that $\omega(v)<1$. One has

$$
\left\|d_{x} d_{s}\right\|_{\infty} \leq\left\|d_{x} d_{s}\right\| \leq\left\|d_{x}\right\|\left\|d_{s}\right\| \leq \frac{1}{2}\left(\left\|d_{x}\right\|^{2}+\left\|d_{s}\right\|^{2}\right)=\omega(v) .
$$

It follows that $\left\|d_{x} d_{s}\right\|_{\infty}<1$. Hence $\omega(v)<1$ implies that the iterates $\left(x^{+}, y^{+}, s^{+}\right)$ are strictly feasible, by Corollary 3.2. We proceed by deriving an upper bound for $\delta\left(x^{+}, s^{+} ; \mu^{+}\right)$. By definition $(2.4)$ we have

$$
\delta\left(x^{+}, s^{+} ; \mu^{+}\right)=\frac{1}{2}\left\|v^{+}-\left(v^{+}\right)^{-1}\right\|, \text { where } v^{+}=\sqrt{\frac{x^{+} s^{+}}{\mu^{+}}} .
$$

In what follows we denote $\delta\left(x^{+}, s^{+} ; \mu^{+}\right)$and briefly by $\delta\left(v^{+}\right)$. We also use the function $\xi$ defined by

$$
\xi(t):=\frac{1+t}{1-\theta}+\frac{1-\theta}{1+t}-2=\frac{(\theta+t)^{2}}{(1-\theta)(1+t)} \geq 0, \quad t>-1 .
$$

LEMMA 3.3. If $\omega(v)<1$, then $4 \delta\left(v^{+}\right)^{2} \leq(n-1) \xi(0)+\max (\xi(\omega(v)), \xi(-\omega(v)))$.

Proof. After dividing both sides in $x^{+} s^{+}=\mu\left(e+d_{x} d_{s}\right)$ by $\mu^{+}$we get

$$
\left(v^{+}\right)^{2}=\frac{\mu\left(e+d_{x} d_{s}\right)}{\mu^{+}}=\frac{e+d_{x} d_{s}}{1-\theta} .
$$

As a consequence we have

$$
4 \delta\left(v^{+}\right)^{2}=-2 n+\sum_{i=1}^{n} \frac{1+d_{x i} d_{s i}}{1-\theta}+\sum_{i=1}^{n} \frac{1-\theta}{1+d_{x i} d_{s i}}=\sum_{i=1}^{n} \xi\left(d_{x i} d_{s i}\right) .
$$

Because of Lemma A.1 this implies that

$$
4 \delta\left(v^{+}\right)^{2} \leq(n-1) \xi(0)+\max (\xi(\omega(v)), \xi(-\omega(v)))
$$

proving the lemma. $\quad$ (

In order to obtain an upper bound for $\omega(v)$ we need to consider the vectors $d_{x}$ and $d_{s}$ in more detail. This is the subject of the next section. 
3.2. Upper bound for $\boldsymbol{\omega}(\boldsymbol{v})$. One may easily check that the system (2.5)-(2.7), which defines the search directions $\Delta x, \Delta y$, and $\Delta s$, can be expressed in terms of the scaled search directions $d_{x}$ and $d_{s}$ as follows:

$$
\begin{aligned}
\bar{A} d_{x} & =\theta \nu r_{b}^{0}, \\
\bar{A}^{T} \frac{\Delta y}{\mu}+d_{s} & =\theta \nu v s^{-1} r_{c}^{0}, \\
d_{x}+d_{s} & =v^{-1}-v,
\end{aligned}
$$

where

$$
\bar{A}=A V^{-1} X, \quad V=\operatorname{diag}(v), \quad X=\operatorname{diag}(x) .
$$

Let us denote the null space of the matrix $\bar{A}$ as $\mathcal{L}$. So, $\mathcal{L}:=\left\{\eta \in \mathbf{R}^{n}: \bar{A} \eta=0\right\}$. Then the affine space $\left\{\eta \in \mathbf{R}^{n}: \bar{A} \eta=\theta \nu r_{b}^{0}\right\}$ equals $d_{x}+\mathcal{L}$. Note that due to a well-known result from linear algebra the row space of $\bar{A}$ equals the orthogonal complement $\mathcal{L}^{\perp}$ of $\mathcal{L}$. Obviously, $d_{s} \in \theta \nu v s^{-1} r_{c}^{0}+\mathcal{L}^{\perp}$. Also note that $\mathcal{L} \cap \mathcal{L}^{\perp}=\{0\}$, and as a consequence the affine spaces $d_{x}+\mathcal{L}$ and $d_{s}+\mathcal{L}^{\perp}$ meet in a unique point. This point is denoted by $q$.

LEMMA 3.4. Let $q$ be the (unique) point in the intersection of the affine spaces $d_{x}+\mathcal{L}$ and $d_{s}+\mathcal{L}^{\perp}$. Then

$$
2 \omega(v) \leq\|q\|^{2}+(\|q\|+2 \delta(v))^{2} .
$$

Proof. To simplify the notation in this proof we denote $r=v^{-1}-v$ and $\delta=$ $\frac{1}{2}\|r\|(=\delta(v))$. Since $\mathcal{L}+\mathcal{L}^{\perp}=\mathbf{R}^{n}$, there exist $q_{1}, r_{1} \in \mathcal{L}$ and $q_{2}, r_{2} \in \mathcal{L}^{\perp}$ such that $q=q_{1}+q_{2}$ and $r=r_{1}+r_{2}$. Since $d_{x}-q \in \mathcal{L}$ implies $d_{x}-q_{2} \in \mathcal{L}$ we have $d_{x}-q_{2}=\ell_{1}$ for some $\ell_{1} \in \mathcal{L}$. Similarly, $d_{s}-q_{1}=\ell_{2}$ for some $\ell_{2} \in \mathcal{L}^{\perp}$. Adding these two relations gives $\ell_{1}+\ell_{2}=d_{x}+d_{s}-\left(q_{1}+q_{2}\right)=r-q$, which implies $\ell_{1}=r_{1}-q_{1}$ and $\ell_{2}=r_{2}-q_{2}$. Substitution gives

$$
d_{x}=q_{2}+\ell_{1}=\left(r_{1}-q_{1}\right)+q_{2}, \quad d_{s}=q_{1}+\ell_{2}=q_{1}+\left(r_{2}-q_{2}\right) .
$$

Since the spaces $\mathcal{L}$ and $\mathcal{L}^{\perp}$ are orthogonal we conclude from this that

$$
2 \omega(v)=\left\|d_{x}\right\|^{2}+\left\|d_{s}\right\|^{2}=\left\|r_{1}-q_{1}\right\|^{2}+\left\|q_{2}\right\|^{2}+\left\|q_{1}\right\|^{2}+\left\|r_{2}-q_{2}\right\|^{2}=\|q-r\|^{2}+\|q\|^{2} .
$$

Due to the triangle inequality we have $\|q-r\| \leq\|q\|+\|r\|=\|q\|+2 \delta$, and hence the lemma follows.

3.3. Upper bound for $\|\boldsymbol{q}\|$. In this section we derive an upper bound for $\|q\|$. Before doing this we recall that our initial iterates $\left(x^{0}, y^{0}, s^{0}\right)$ are chosen in the usual way. So, we assume that $\zeta>0$ is such that $\left\|x^{*}+s^{*}\right\|_{\infty} \leq \zeta$ for some optimal solutions $x^{*}$ of $(\mathrm{P})$ and $\left(y^{*}, s^{*}\right)$ of (D), and we start the algorithm with

$$
x^{0}=s^{0}=\zeta e, \quad y^{0}=0, \quad \mu^{0}=\zeta^{2} .
$$

In the proof of the lemma below we use the following easy consequences of these assumptions:

$$
0 \leq x^{0}-x^{*} \leq \zeta e, \quad 0 \leq s^{0}-s^{*} \leq \zeta e .
$$

Copyright $@$ by SIAM. Unauthorized reproduction of this article is prohibited. 
LEMMA 3.5. One has $\|q\| \leq \theta \frac{\|x\|_{1}+\|s\|_{1}}{\zeta \min (v)}$.

Proof. From the definition (3.6) of $\bar{A}$ we deduce that $\bar{A}=\sqrt{\mu} A D$, where

$$
D=\operatorname{diag}\left(\frac{x v^{-1}}{\sqrt{\mu}}\right)=\operatorname{diag}\left(\sqrt{\frac{x}{s}}\right)=\operatorname{diag}\left(\sqrt{\mu} v s^{-1}\right) .
$$

Since $q$ satisfies $(3.3)$ (with $d_{x}=q$ ) and

$$
r_{b}^{0}=b-A x^{0}=A\left(x^{*}-x^{0}\right)=\frac{1}{\sqrt{\mu}} \bar{A} D^{-1}\left(x^{*}-x^{0}\right),
$$

we obtain $q-\frac{\theta \nu}{\sqrt{\mu}} D^{-1}\left(x^{*}-x^{0}\right) \in \mathcal{L}$. On the other hand, using that $q$ satisfies (3.4) (with $d_{s}=q$ ) one proves in a similar way that that $q-\frac{\theta \nu}{\sqrt{\mu}} D\left(s^{*}-s^{0}\right) \in \mathcal{L}^{\perp}$. These two properties of $q$ imply that

$$
\|q\| \leq \frac{\theta \nu}{\sqrt{\mu}} \sqrt{\left\|D\left(s^{*}-s^{0}\right)\right\|^{2}+\left\|D^{-1}\left(x^{*}-x^{0}\right)\right\|^{2}} .
$$

Using (3.8) and the definition of $D$, we obtain

$$
\|q\| \leq \frac{\theta \nu \zeta}{\sqrt{\mu}} \sqrt{\|D e\|^{2}+\left\|D^{-1} e\right\|^{2}}=\frac{\theta \nu \zeta}{\sqrt{\mu}} \sqrt{e^{T}\left(\frac{x}{s}+\frac{s}{x}\right)} .
$$

One has

$$
\begin{aligned}
e^{T}\left(\frac{x}{s}+\frac{s}{x}\right) & =e^{T} \frac{x^{2}+s^{2}}{x s}=e^{T} \frac{x^{2}+s^{2}}{\mu v^{2}} \leq \frac{\|x\|^{2}+\|s\|^{2}}{\mu \min (v)^{2}} \\
& \leq\left(\frac{\|x\|+\|s\|}{\sqrt{\mu} \min (v)}\right)^{2} \leq\left(\frac{\|x\|_{1}+\|s\|_{1}}{\sqrt{\mu} \min (v)}\right)^{2} .
\end{aligned}
$$

Substituting this into (3.9), also using $\mu=\mu^{0} \nu=\nu \zeta^{2}$, we obtain the inequality in the lemma.

In the next section we derive an upper bound for $\|x\|_{1}+\|s\|_{1}$.

3.4. Upper bound for $\|x\|_{1}+\|s\|_{1}$. Due to our choice of the optimal solutions $x^{*}$ and $\left(y^{*}, s^{*}\right)$ and the definition of $\zeta($ see $(1.1))$ we have

$$
A x^{*}=b, \quad A^{T} y^{*}+s^{*}=c, \quad x^{*} s^{*}=0,
$$

where $0 \leq x^{*} \leq \zeta e$ and $0 \leq s^{*} \leq \zeta e$. On the other hand we have

$$
b-A x=\nu(b-A \zeta e), \quad c-A^{T} y-s=\nu(c-\zeta e),
$$

where $x \geq 0$ and $s \geq 0$. Replacing $b$ by $A x^{*}$ and $c$ by $A^{T} y^{*}+s^{*}$ we get

$$
A x^{*}-A x=\nu\left(A x^{*}-A \zeta e\right), \quad A^{T} y^{*}+s^{*}-A^{T} y-s=\nu\left(A^{T} y^{*}+s^{*}-\zeta e,\right.
$$

which implies

$$
A\left(x^{*}-x-\nu x^{*}+\nu \zeta e\right)=0, \quad A^{T}\left(y^{*}-y-\nu y^{*}\right)=s-s^{*}+\nu s^{*}-\nu \zeta e .
$$

Using again that the row space of a matrix and its null space are orthogonal, we obtain

$$
\left[(1-\nu) x^{*}+\nu \zeta e-x\right]^{T}\left[(1-\nu) s^{*}+\nu \zeta e-s\right]=0
$$

Copyright (C) by SIAM. Unauthorized reproduction of this article is prohibited. 
Hence, defining $a:=(1-\nu) x^{*}+\nu \zeta e$ and $b:=(1-\nu) s^{*}+\nu \zeta e$, we have $(a-x)^{T}$ $(b-s)=0$. This gives

$$
a^{T} b+x^{T} s=a^{T} s+b^{T} x .
$$

Since $x^{* T} s^{*}=0$ and $x^{*}+s^{*} \leq \zeta e$, we may write

$$
\begin{aligned}
a^{T} b+x^{T} s & =\left[(1-\nu) x^{*}+\nu \zeta e\right]^{T}\left[(1-\nu) s^{*}+\nu \zeta e\right]+x^{T} s \\
& =\nu(1-\nu)\left(x^{*}+s^{*}\right)^{T} \zeta e+\nu^{2} \zeta^{2} n+x^{T} s \\
& \leq \nu(1-\nu)(\zeta e)^{T} \zeta e+\nu^{2} \zeta^{2} n+x^{T} s=\nu \zeta^{2} n+x^{T} s .
\end{aligned}
$$

Since $a^{T} s \geq \nu \zeta e^{T} x$ and $b^{T} x \geq \nu \zeta e^{T} s$, we also have

$$
a^{T} s+b^{T} x \geq \nu \zeta e^{T}(x+s)=\nu \zeta\left(\|x\|_{1}+\|s\|_{1}\right) .
$$

Hence, also using $x^{T} s=\mu e^{T} v^{2}=\mu\|v\|^{2}$ we obtain

$$
\nu \zeta\left(\|x\|_{1}+\|s\|_{1}\right) \leq \nu \zeta^{2} n+x^{T} s=\nu \zeta^{2} n+\mu\|v\|^{2} .
$$

Since $\mu=\nu \zeta^{2}$ this simplifies to $\|x\|_{1}+\|s\|_{1} \leq \zeta\left(n+\|v\|^{2}\right)$. Substitution into Lemma 3.5 yields that

$$
\|q\| \leq \theta \frac{\|x\|_{1}+\|s\|_{1}}{\zeta \min (v)} \leq \frac{\theta\left(n+\|v\|^{2}\right)}{\min (v)} .
$$

One easily checks that if $\delta=\delta(v)$ is given, then $\|v\|$ is maximal if $v \geq e$ and all elements of $v$ are equal to $\delta / \sqrt{n}+\sqrt{1+\delta^{2} / n}$. Therefore,

$$
\|v\|^{2} \leq n\left(\frac{\delta}{\sqrt{n}}+\sqrt{1+\frac{\delta^{2}}{n}}\right)^{2}=n+2 \delta^{2}+2 \delta \sqrt{n+\delta^{2}} .
$$

By Lemma 2.2 one has $\min (v) \geq-\delta+\sqrt{1+\delta^{2}}$. Substitution of these two bounds into (3.10) yields

$$
\|q\| \leq \frac{2 \theta\left(n+\delta^{2}+\delta \sqrt{n+\delta^{2}}\right)}{-\delta+\sqrt{1+\delta^{2}}}
$$

3.5. Values for $\boldsymbol{\theta}$ and $\tau$. Our aim is to find a positive number $\tau$ such that if $\delta(v) \leq \tau$ holds, then $\delta\left(v^{+}\right) \leq \tau$. By Lemma 3.3 we have $4 \delta\left(v^{+}\right)^{2} \leq(n-1) \xi(0)+$ $\max (\xi(\omega), \xi(-\omega))$, provided that $\omega:=\omega(v)<1$. According to Lemma 3.4 we have $2 \omega \leq\|q\|^{2}+(\|q\|+2 \delta(v))^{2}$, where $\|q\|$ is bounded from above as in (3.11). Hence, it suffices if $\tau$ is such that the three inequalities

$$
\begin{aligned}
0 \leq \delta & \leq \tau, \\
\omega & \leq \frac{1}{2}\left(\|q\|^{2}+(\|q\|+2 \delta)^{2}\right), \\
\|q\| & \leq \frac{2 \theta\left(n+\delta^{2}+\delta \sqrt{n+\delta^{2}}\right)}{-\delta+\sqrt{1+\delta^{2}}}
\end{aligned}
$$

imply the inequalities $\omega<1$ and

$$
\frac{1}{2} \sqrt{(n-1) \xi(0)+\max (\xi(\omega), \xi(-\omega))} \leq \tau .
$$

Copyright (c) by SIAM. Unauthorized reproduction of this article is prohibited. 
In the rest of this section we show that this implication holds if $\theta$ and $\tau$ are taken as follows:

$$
\theta=\frac{1}{8 n}, \quad \tau=\frac{1}{5}
$$

One easily verifies that the right-hand-side expression in (3.14) is monotonically increasing with respect to $\delta$. Hence, using (3.12) and (3.14) we obtain

$$
\|q\| \leq \frac{2 \theta n\left(1+\frac{\delta^{2}}{n}+\frac{\delta}{\sqrt{n}} \sqrt{1+\frac{\delta^{2}}{n}}\right)}{-\delta+\sqrt{1+\delta^{2}}} \leq \frac{1+\frac{\tau^{2}}{n}+\frac{\tau}{\sqrt{n}} \sqrt{1+\frac{\tau^{2}}{n}}}{4\left(-\tau+\sqrt{1+\tau^{2}}\right)} .
$$

Note that the last expression decreases when $n$ increases. Therefore the same holds for the function $h(n, \tau)$ defined by

$$
h(n, \tau):=\frac{1}{2}\left[\left(\frac{1+\frac{\tau^{2}}{n}+\frac{\tau}{\sqrt{n}} \sqrt{1+\frac{\tau^{2}}{n}}}{4\left(-\tau+\sqrt{1+\tau^{2}}\right)}\right)^{2}+\left(\frac{1+\frac{\tau^{2}}{n}+\frac{\tau}{\sqrt{n}} \sqrt{1+\frac{\tau^{2}}{n}}}{4\left(-\tau+\sqrt{1+\tau^{2}}\right)}+2 \tau\right)^{2}\right],
$$

which is an upper bound for $\omega$, by (3.13). In order to proceed it is convenient to introduce the function

$$
\chi(t):=\max (\xi(t), \xi(-t)), \quad 0 \leq t<1 .
$$

We claim that $\chi(t)$ is increasing for $t \geq 0$. This follows from the fact that $\xi(t)$ is convex (because $\left.\xi^{\prime \prime}(t)=\frac{2(1-\theta)}{(1+t)^{3}}>0\right)$. But then $\xi(-t)$ is also convex. As a consequence $\chi(t)$ is convex. Since $\chi(t)$ is also symmetric with respect to the origin it follows that $\chi(t)$ is increasing for $t \geq 0$, proving the claim. hold if

Hence, $0 \leq \omega \leq h(n, \tau)$ implies $\chi(\omega) \leq \chi(h(n, \tau))$. Therefore, (3.15) will certainly

$$
\frac{1}{2} \sqrt{(n-1) \xi(0)+\chi(h(n, \tau))} \leq \tau
$$

One has

$$
(n-1) \xi(0)=(n-1)\left(\frac{1}{1-\theta}+(1-\theta)-2\right)=\frac{(n-1) \theta^{2}}{1-\theta}=\frac{n-1}{8 n(8 n-1)},
$$

which makes clear that $(n-1) \xi(0)$ is decreasing in $n$. We already established that $h(n, \tau)$ is decreasing in $n$, while $\chi(t)$ is increasing in $t$. This implies that $\chi(h(n, \tau))$ also decreases if $n$ increases. We conclude that if (3.18) is satisfied for $n=2$, then it is certainly satisfied for all larger values of $n$. Hence it suffices if $\tau$ satisfies

$$
h(2, \tau)<1, \quad \frac{1}{2} \sqrt{\frac{1}{16 \cdot 15}+\chi(h(2, \tau))} \leq \tau .
$$

These are inequalities in $\tau$ alone. For $\tau=\frac{1}{5}$ one has $h(2, \tau)=0.347587<1$. Defining

$$
g_{+}(\tau):=\frac{1}{2} \sqrt{\frac{1}{16 \cdot 15}+\xi(h(2, \tau))}, \quad g_{-}(\tau):=\frac{1}{2} \sqrt{\frac{1}{16 \cdot 15}+\xi(-h(2, \tau))},
$$

we may conclude that inequality (3.19) will hold if the inequalities $g_{+}(\tau) \leq \tau$ and $g_{-}(\tau) \leq \tau$ are valid. It turns out that $g_{+}\left(\frac{1}{5}\right)=0.180162$ and $g_{-}\left(\frac{1}{5}\right)=0.174747$. Hence we may state the following result without further proof.

Lemma 3.6. If $\theta$ and $\tau$ are given by (3.16), then $\delta(v) \leq \tau$ implies $\delta\left(v^{+}\right) \leq \tau$. 
3.6. Complexity analysis. We have found that if $n \geq 2$ and at the start of an iteration the iterates satisfy $\delta(x, s ; \mu) \leq \tau$, and $\tau$ and $\theta$ are as defined in (3.16), then after the Newton step the new iterates satisfy $\delta\left(x^{+}, s^{+} ; \mu^{+}\right) \leq \tau$. This makes the algorithm well defined.

In each iteration the norms of the residual vectors are reduced by the factor $1-\theta$ and the same holds for the barrier parameter $\mu$ (whose initial value equals $n \zeta^{2}$ ). It is now well known (see, e.g., [10]) that then the total number of main iterations is bounded above by

$$
\frac{1}{\theta} \log \frac{\max \left\{n \zeta^{2},\left\|r_{b}^{0}\right\|,\left\|r_{c}^{0}\right\|\right\}}{\varepsilon} .
$$

Since $\theta=1 /(8 n)$, this yields the following result.

Theorem 3.7. Let (P) and (D) be feasible and $\zeta>0$ such that $\left\|x^{*}+s^{*}\right\|_{\infty} \leq \zeta$ for some optimal solutions $x^{*}$ of $(\mathrm{P})$ and $\left(y^{*}, s^{*}\right)$ of $(\mathrm{D})$. Then after at most

$$
8 n \log \frac{\max \left\{n \zeta^{2},\left\|r_{b}^{0}\right\|,\left\|r_{c}^{0}\right\|\right\}}{\varepsilon}
$$

inner iterations the algorithm finds an $O(\varepsilon)$-solution of $(\mathrm{P})$ and $(\mathrm{D})$.

It is worth noting that this result improves the iteration bound in [10, Theorem 4.8] with a factor $2 \sqrt{2} .^{1}$ We refer to [10] for a discussion on how to choose the number $\zeta$ in the algorithm and on how infeasibility and/or unboundedness of the problems (P) and (D) can be established with the algorithm presented in this paper.

4. Concluding remarks. The method presented in this paper is simpler than the method in [10]. The earlier method used in each main iteration a so-called feasibility step and three centering steps, whereas the new algorithm does not need the centering steps. The analysis is also simpler and the iteration bound is improved by a factor $2 \sqrt{2}$. This improvement has been achieved by a much tighter estimate of the proximity measure $\delta\left(x^{+}, s^{+} ; \mu^{+}\right)$after a feasibiliy step; the tighter estimate is due to a new lemma, Lemma A.1 in the appendix, which might also be useful in the analysis of other methods that are based on the proximity measure used in this paper. Finally it might be emphasized that the iteration bound in this paper is a worst-case bound, as is usual for theoretical iteration bounds for IPMs (including IIPMs). When solving a particular problem, usually much smaller iteration numbers can be realized by taking $\theta$ larger than the value that is theoretically justified.

Appendix A. Fundamental inequality. In this appendix we prove the following lemma, where the function $\xi$ is as defined in (3.2).

Lemma A.1. Let $a, b \in \mathbf{R}^{n}$ and $f(a, b):=\sum_{i=1}^{n} \xi\left(a_{i} b_{i}\right)$. If $\|a\|^{2}+\|b\|^{2} \leq 2 r^{2}$, with $r \in[0,1)$, then

$$
f(a, b) \leq(n-1) \xi(0)+\max \left(\xi\left(r^{2}\right), \xi\left(-r^{2}\right)\right) .
$$

Proof. To start with, let us maximize $f(a, b)$ subject to the condition that $\|a\|^{2}+$ $\|b\|^{2}=2 r^{2}$ with $r \in(0,1)$. So we consider the problem

$$
\max \left\{\sum_{i=1}^{n}\left[\frac{1+a_{i} b_{i}}{1-\theta}+\frac{1-\theta}{1+a_{i} b_{i}}-2\right]:\|a\|^{2}+\|b\|^{2}=2 r^{2}\right\} .
$$

\footnotetext{
${ }^{1}$ Let us also point out that in [10, Conjecture 5.1] it was conjectured that the iteration bound in [10, Theorem 4.8] could be improved by a factor $\sqrt{2 n}$. This conjecture has been shown to be false in $[2]$.
} 
The first order optimality conditions for this problem are

$$
\frac{b_{i}}{1-\theta}-\frac{(1-\theta) b_{i}}{\left(1+a_{i} b_{i}\right)^{2}}=2 \lambda a_{i}, \quad \frac{a_{i}}{1-\theta}-\frac{(1-\theta) a_{i}}{\left(1+a_{i} b_{i}\right)^{2}}=2 \lambda b_{i}, \quad 1 \leq i \leq n,
$$

where $\lambda$ is a Lagrange multiplier. By subtracting and adding these two relations it follows that

$$
b_{i}=a_{i} \quad \text { or } \quad \frac{1}{1-\theta}-\frac{1-\theta}{\left(1+a_{i} b_{i}\right)^{2}}=-2 \lambda, \quad 1 \leq i \leq n,
$$

and

$$
b_{i}=-a_{i} \quad \text { or } \quad \frac{1}{1-\theta}-\frac{1-\theta}{\left(1+a_{i} b_{i}\right)^{2}}=2 \lambda, \quad 1 \leq i \leq n .
$$

We claim that if the pair $(a, b)$ is optimal then there is no index $i$ such that the second equations in (A.1) and (A.2) are both satisfied. Otherwise we would have $\lambda=0$, and this would imply $\left(1+a_{i} b_{i}\right)^{2}=(1-\theta)^{2}$. The latter gives either $a_{i} b_{i}=-\theta$ or $a_{i} b_{i}=\theta-2<-1$. If $a_{i} b_{i}<-1$, then $a_{i}^{2}+b_{i}^{2} \geq-2 a_{i} b_{i}>2$, which contradicts $r<1$. On the other hand, if $a_{i} b_{i}=-\theta$, then the contribution of the $i$ th term to the objective function is $\xi(-\theta)=0$. This is certainly not optimal, because replacing $b_{i}$ by $-b_{i}$, we get $a_{i} b_{i}=\theta$, and $\xi(\theta)>0$. Hence the claim follows. As a consequence we must have $b_{i}= \pm a_{i}$ for each $i$.

Without loss of generality we may assume $a \geq 0$, because if the pair $\left(a_{i}, b_{i}\right)$ occurs in an optimal solution, the pair $\left(-a_{i},-b_{i}\right)$ is also optimal. Defining

$$
I_{+}=\left\{i: b_{i}=a_{i}\right\}, \quad I_{-}=\left\{i: b_{i}=-a_{i}\right\},
$$

we claim that if the pair $(a, b)$ is optimal, then $\left|I_{+}\right| \leq 1$. This goes as follows. Suppose that $\left|I_{+}\right|>1$. Then there exist two different indices $i_{1}, i_{2}$ in $I_{+}$. Since $b_{i}=a_{i}$ if $i \in I_{+}$, we deduce from the second equation in (A.2) that $a_{i_{1}}=a_{i_{2}}$. We denote this common value as $\alpha$. Now let the pair $\left(a^{\prime}, b^{\prime}\right)$ arise from the pair $(a, b)$ by replacing $a_{i_{1}}$ and $b_{i_{1}}$ by $\alpha \sqrt{2}$, and $a_{i_{2}}$ and $b_{i_{2}}$ by 0 . Then it is clear $\left\|a^{\prime}\right\|^{2}+\left\|b^{\prime}\right\|^{2}=\|a\|^{2}+\|b\|^{2}=2 r^{2}$. Moreover, one has $f\left(a^{\prime}, b^{\prime}\right)-f(a, b)=\xi\left(2 \alpha^{2}\right)+\xi(0)-2 \xi\left(\alpha^{2}\right)$. Hence we may write

$$
\begin{aligned}
f\left(a^{\prime}, b^{\prime}\right)-f(a, b)= & {\left[\frac{1+2 \alpha^{2}}{1-\theta}+\frac{1-\theta}{1+2 \alpha^{2}}-2\right]+\left[\frac{1}{1-\theta}+\frac{1-\theta}{1}-2\right] } \\
& -2\left[\frac{1+\alpha^{2}}{1-\theta}+\frac{1-\theta}{1+\alpha^{2}}-2\right] \\
= & \frac{1-\theta}{1+2 \alpha^{2}}+1-\theta-2 \frac{1-\theta}{1+\alpha^{2}}=(1-\theta)\left[\frac{2\left(1+\alpha^{2}\right)}{1+2 \alpha^{2}}-\frac{2}{1+\alpha^{2}}\right] \\
= & 2(1-\theta) \frac{\left(1+\alpha^{2}\right)^{2}-\left(1+2 \alpha^{2}\right)}{\left(1+2 \alpha^{2}\right)\left(1+\alpha^{2}\right)}=2(1-\theta) \frac{\alpha^{4}}{\left(1+2 \alpha^{2}\right)\left(1+\alpha^{2}\right)}>0 .
\end{aligned}
$$

This proves that the pair $(a, b)$ is not a global maximizer if $\left|I_{+}\right|>1$, which justifies our claim that $\left|I_{+}\right| \leq 1$. By replacing $\alpha^{2}$ by $-\alpha^{2}$ in the above arguments one obtains in exactly the same way that also $\left|I_{-}\right| \leq 1$.

It remains to deal with the case where $a$ has at most two positive entries, $a_{i_{1}}$ and $a_{i_{2}}$, say, and the corresponding entries of $b$ are $a_{i_{1}}$ and $-a_{i_{2}}$, respectively. To 
simplify the notation we neglect for the moment the indices $i$ for which $a_{i}=0$ by taking $a=(\alpha, \beta)$, and $b=(\alpha,-\beta)$, with $\alpha$ and $\beta$ nonnegative, whereas $\|a\|^{2}+\|b\|^{2}=$ $2\left(\alpha^{2}+\beta^{2}\right)=2 r^{2}$. Hence $r=\sqrt{\alpha^{2}+\beta^{2}}$. As a consequence we may write

$$
\alpha=r \cos (\varphi), \quad \beta=r \sin (\varphi) \text { for some } \varphi \in\left[0, \frac{\pi}{2}\right] .
$$

We then have $f(a, b)=g(\varphi)$, where $g(\varphi)=\xi\left(r^{2} \cos ^{2} \varphi\right)+\xi\left(-r^{2} \sin ^{2} \varphi\right)$. Using $\xi^{\prime}(x)=$ $1 /(1-\theta)-(1-\theta) /(1+x)^{2}$, one easily verifies that

$$
g^{\prime}(\varphi)=r^{2} \sin (2 \varphi)\left[\frac{-2}{1-\theta}+\frac{1-\theta}{\left(1+r^{2} \cos ^{2} \varphi\right)^{2}}+\frac{1-\theta}{\left(1-r^{2} \sin ^{2} \varphi\right)^{2}}\right] .
$$

We see that $g^{\prime}(\varphi)=0$ at the boundary values for $\varphi$ (i.e., $\varphi=0$ and $\varphi=\pi / 2$ ), because of the factor $\sin (2 \varphi)$. So $g(\varphi)$ has stationary points at $\varphi=0$ and $\varphi=\pi / 2$ and may be also at a point where the expression between brackets vanishes. The value at $\varphi=0$ of the bracketed expression is

$$
\frac{-2}{1-\theta}+\frac{1-\theta}{\left(1+r^{2}\right)^{2}}+1-\theta
$$

which is certainly negative. The bracketed expression is strictly increasing between the boundary values of $\varphi$, because its derivative with respect to $\varphi$ is given by

$$
2 r^{2}(1-\theta) \sin (2 \varphi)\left[\frac{1}{\left(1+r^{2} \cos ^{2} \varphi\right)^{3}}+\frac{1}{\left(1-r^{2} \sin ^{2} \varphi\right)^{3}}\right],
$$

which is positive. It follows that $g(\varphi)$ has at most one stationary point in the interval $\left(0, \frac{\pi}{2}\right)$.

In order to proceed we compute the second derivative $g^{\prime \prime}(\varphi)$. This is given by

$$
\begin{aligned}
g^{\prime \prime}(\varphi)= & \frac{2 r^{2}}{1-\theta}\left[-2 \cos (2 \varphi)+\frac{(1-\theta)^{2} \cos (2 \varphi)}{\left(1+r^{2} \cos ^{2} \varphi\right)^{2}}+\frac{(1-\theta)^{2} \cos (2 \varphi)}{\left(1-r^{2} \sin ^{2} \varphi\right)^{2}}\right. \\
& \left.+\frac{r^{2}(1-\theta)^{2} \sin (2 \varphi)^{2}}{\left(1+r^{2} \cos ^{2} \varphi\right)^{3}}+\frac{r^{2}(1-\theta)^{2} \sin (2 \varphi)^{2}}{\left(1-r^{2} \sin ^{2} \varphi\right)^{3}}\right]
\end{aligned}
$$

Hence we have

$$
g^{\prime \prime}(0)=\frac{2 r^{2}}{1-\theta}\left[-2+\frac{(1-\theta)^{2}}{\left(1+r^{2}\right)^{2}}+(1-\theta)^{2}\right] .
$$

We see that $g^{\prime \prime}(0)<0$. Since $g^{\prime}(0)=0$, it follows that $g(\varphi)$ is decreasing for small positive values of $\varphi$. If there is no stationary point in the interval $\left(0, \frac{\pi}{2}\right)$, then $g(\varphi)$ is decreasing on the whole interval $\left(0, \frac{\pi}{2}\right)$, which implies that $g(0)$ is the global maximum value. On the other hand, if there is a stationary point in the interval $\left(0, \frac{\pi}{2}\right)$, then since there is at most one such point, the only candidates for the maximizing values of $\varphi$ are the boundary points $\varphi=0$ and $\varphi=\frac{\pi}{2}$. Then we have either $\beta=0$ or $\alpha=0$.

We conclude that if $(a, b)$ maximizes $f(a, b)$, then $a_{i}$ is positive for at most one index $i$, and $a_{i} b_{i}= \pm r^{2}$. Also taking into account the indices for which $a_{i}=0$, we conclude that the global maximum value of $g(\varphi)$ is equal to one of the following 
two values: $\xi\left(r^{2}\right)+(n-1) \xi(0)$ or $\xi\left(-r^{2}\right)+(n-1) \xi(0)$. This proves the lemma if $\|a\|^{2}+\|b\|^{2}=2 r^{2}$.

Finally we have to deal with the case where $\|a\|^{2}+\|b\|^{2}=2 \varrho^{2} \leq 2 r^{2}$. In that case the above proof implies

$$
f(a, b) \leq(n-1) \xi(0)+\max \left(\xi\left(\varrho^{2}\right), \xi\left(-\varrho^{2}\right)\right)=(n-1) \xi(0)+\chi\left(\xi\left(\varrho^{2}\right)\right),
$$

where $\chi(t)$ is the function defined in (3.17). As we showed there, $\chi(t)$ is increasing for $t \geq 0$. Hence the statement in the lemma follows.

Acknowledgment. Thanks are due to Guoyong Gu (Nanjing University, China) and three anonymous referees for their careful reading of a previous version; their valuable comments helped to increase the readability of the paper. I would also like to express my thanks to the associate editor, Nick Gould, for the way he managed the reviewing process.

\section{REFERENCES}

[1] R. M. Freund, A potential-function reduction algorithm for solving a linear program directly from an infeasible 'warm start,' Math. Program., 52 (1991), pp. 441-466.

[2] G. Gu And C. Roos, Counterexample to a conjecture on an infeasible interior-point method, SIAM J. Optim., 20 (2010), pp. 1862-1867.

[3] B. KheIRFAm, Simplified infeasible interior-point algorithm for SDO using full Nesterov-Todd step, Numer. Algorithms, 59 (2012), pp. 589-606.

[4] M. Kojima, S. Mizuno, And A. Yoshise, A little theorem of the big $M$ in interior point algorithms, Math. Program., 59 (1993), pp. 361-375.

[5] Z. LiU AND W. Sun, An infeasible interior-point algorithm with full-Newton step for linear optimization, Numer. Algorithms, 46 (2007), pp. 173-188.

[6] H. MANSOURI, Full-Newton step infeasible interior-point algorithm for SDO problems, Kybernetika, 48 (2012), pp. 907-923.

[7] S. Mizuno, M. J. Todd, And Y. Ye, A surface of analytic centers and infeasible-interior-point algorithms for linear programming, Math. Oper. Res., 20 (1995), pp. 135-162.

[8] R. D. C. Monteiro And I. Adlen, Interior-path following primal-dual algorithms: Part I: Linear programming, Math. Program., 44 (1989), pp. 27-41.

[9] F. A. Potra, An infeasible-interior-point predictor-corrector algorithm for linear programming, SIAM J. Optim., 6 (1996), pp. 19-32.

[10] C. Roos, A full-Newton step $O(n)$ infeasible interior-point algorithm for linear optimization, SIAM J. Optim., 16 (2006), pp. 1110-1136.

[11] C. Roos, T. Terlaky, And J.-Ph. Vial, Theory and Algorithms for Linear Optimization, Springer, Chichester, UK, 2005.

[12] S. J. Wright, Primal-Dual Interior-Point Methods, SIAM, Philadelphia, 1996.

[13] Y. Ye, M. J. Todd, And S. Mizuno, An $O(\sqrt{n} L)$-iteration homogeneous and self-dual linear programming algorithm, Math. Oper. Res., 19 (1994), pp. 53-67.

[14] Y. ZhanG, On the convergence of a class of infeasible-interior-point methods for the horizontal linear complementarity problem, SIAM J. Optim., 4 (1994), pp. 208-227.

Copyright $@$ by SIAM. Unauthorized reproduction of this article is prohibited. 